BULL. AUSTRAL. MATH. SOC.

$16 A 42,16 A 36$

VOL. 8 (1973), 159-160.

\title{
On matrix methods in ring theory
}

\section{George Ivanov}

Several classes of rings are studied and their structures determined by a direct method of representing rings as matrix rings. This method is one of the main contributions of the thesis. It is developed in a general form in Chapter 1.

Nonsingular rings with essential socles are investigated in Chapter 2. The main result is a characterization of these rings by an embedding into products of matrix rings over sfields and their bi-vector spaces. When the identity of the ring is finite (that is, a sum of orthogonal primitive idempotents) the embediding is onto and gives the known representation for such a ring. These results are used in Chapter 3 to determine the structure of nonsingular QF-3 rings with finite identities. It is also shown that a nonsingular left, and right, $Q F-3$ ring has a finite identity and finite dimensional left, and right, socles which are essential.

Chapter 4 is devoted to rings whose ideals are quasi-injective (Q-rings). It proves that apart from the simple Artinian rings there is only one class of indecomposable non-local Q-rings. These are the rings of all $m \times m$-matrices, $m$ an integer greater than one, whose non-zero entries are arbitrary elements of a sfield $D$ along the diagonal and arbitrary elements of a null $D$-algebra $V$, whose left and right $D$-dimensions are both equal to one, at the places $(2,1), \ldots,(m, m-I)$ and $(1, m)$.

These four chapters emphasize the dependence of the complexity of a ring's structure on its idempotents. It is the euthor's opinion that a theory of "large" rings could be developed on the structure of their sets of idempotents. This might provide a useful alternative to the traditional

Received 23 November 1972. Thesis submitted to the Australian National University, July 1972. Degree approved, October 1972.

Supervisors: The late Professor Hanna Neumann, Dr Carlton Christensen. 159 
approach to studying rings by placing radical-type restrictions.

The last chapter, Chapter 5 , is in some senses the most important. It determines the structure of left generalized unfserial rings and shows that it is possible, in non-trivial cases, to construct a ring from its ideal lattice. 